\title{
Resonance linewidth and inhomogeneous broadening in a metamaterial array
}

\author{
S. D. Jenkins and J. Ruostekoski \\ School of Mathematics and Centre for Photonic Metamaterials, University of Southampton, Southampton SO17 1BJ, United Kingdom
}

(Received 12 September 2012; published 26 November 2012)

\begin{abstract}
We examine the effect of inhomogeneous broadening on the collective response of a planar metamaterial consisting of asymmetric split-ring resonators. We show that such a response leads to a transmission resonance that can persist when the broadening of individual meta-atom resonance frequencies is roughly one-half the frequency characterizing the split-ring asymmetry. We also find that larger degrees of inhomogeneous broadening can drastically alter the cooperative response, destroying this resonance. The reduced effect of cooperative response due to inhomogeneous broadening may find applications in producing metamaterial samples that more closely mimic homogeneous magnetodielectric medium with well-defined susceptibility and permittivity.
\end{abstract}

DOI: 10.1103/PhysRevB.86.205128

PACS number(s): 78.67.Pt, 42.25.Bs, 41.20.Jb

\section{INTRODUCTION}

There exists increasing experimental evidence that metamaterial samples, consisting of arrays of subwavelengthscale nanostructured circuit elements, can be prepared in the limit where collective interactions between the resonators play a vital role in determining their electromagnetic (EM) responses. For example, experiments on two-dimensional (2D) arrays of closely spaced asymmetric split-ring (ASR) metamolecules have indicated the presence of a high-quality transmission resonance, with a dramatic sensitivity of the resonance linewidth to the number of ASRs in the sample. ${ }^{1}$ The transmission resonance was also observed to depend strongly on the spatial distribution of the mutually interacting resonators. Where a sufficiently large, regular array of ASRs exhibits a high-quality transmission resonance, ${ }^{1}$ introducing disorder in the elements' positions destroys the observed spectral transmission window, ${ }^{2,3}$ further indicating the role collective EM interactions can play in metamaterial dynamics. We recently showed ${ }^{4,5}$ that closely spaced ASR metamolecules interacting via a resonant EM field exhibit collective eigenmodes with strongly suppressed resonance linewidths. The cooperative response yields the characteristic feature in the experimentally observed enhanced quality factor of the transmission resonance in Ref. 1. Numerically analyzing the properties of collective modes with narrow radiative resonance linewidths provided a physical explanation of this phenomenon with an excellent agreement between the simulations and the measurements.

In our previous study ${ }^{5}$ showing how the transmission resonance observed by Fedotov et al. ${ }^{1}$ depends on the linewidth of a particular collective mode, all unit-cell resonators were assumed to respond to EM fields identically. In the preparation of metamaterial samples, fabrication defects, however, may in general lead to variation in the geometry of individual resonators. The current oscillations supported by the unit-cell resonators would therefore possess slightly different resonance frequencies, resulting in inhomogeneously broadened metamaterial arrays. Inhomogeneous broadening changes the conditions of the resonant interaction processes. This may impair the collective, coherent phenomena which are potentially important in several applications and physical effects of metamaterials such as lasing, ${ }^{6}$ generation of highly coherent and directed light beam, ${ }^{7}$ and providing precise control and manipulation of EM fields on a subwavelength scale, as theoretically proposed in Ref. 8 and experimentally observed in Ref. 9 (for other related studies, see for example Ref. 10).

In this work, we study how uncontrolled inhomogeneous broadening of plasmonic resonators limits the observation of collective phenomena in metamaterials. We will examine under what conditions these collective effects can still persist and potential implications of inhomogeneous broadening on metamaterial applications. In particular, we consider a rectangular 2D array of ASR metamolecules, the resonance frequencies of which are stochastically varied. We numerically evaluate ensemble averages of the EM response of the metamaterial over the stochastic distributions of the resonator properties. These show how increased inhomogeneous broadening inhibits the response of the coherent collective modes responsible for the transmission resonance observed in Ref. 1. Moreover, we find the effects of interactions between different discrete resonator elements, which result in the narrowing of the radiative resonance linewidth, are diminished as a function of an increasing inhomogeneous broadening. Our results therefore illustrate how maintaining uniformity in the fabrication process is essential in designing new metamaterial-based devices, the applications of which rely on strong interactions between the resonator elements and on a cooperative response.

The multiple-scale spatial structure associated with nanofabricated resonators in metamaterial arrays, along with the wave nature of scattered EM fields, poses a theoretical challenge for studies of the response of these systems to resonant EM fields. Interactions resulting from recurrent scattering events, in which a field is scattered more than once by the same resonator, frequently play a crucial role in the cooperative system responses., ${ }^{4,51-22}$ While in an infinite, regular lattice the translational symmetry can be exploited to calculate approximate local field corrections in a medium of discrete scatterers, ${ }^{23}$ recurrent scattering processes are generally more difficult to model in finite-sized systems with complex geometries.

However, since inhomogeneous broadening reduces the cooperative effects arising from recurrent scattering, engineering a controlled amount of inhomogeneous broadening into the metamaterial, e.g., via geometrical variation of the resonators, may provide a practical means to produce samples 
that are easier to design and theoretically analyze. In particular, we find that with an increasing inhomogeneous broadening, the response of the system approaches that of standard continuous medium electrodynamics. Reducing cooperative effects is potentially important because several metamaterial applications, such as diffraction-free lenses formed from a medium with a negative refractive index, ${ }^{24-26}$ are simplest to realize with a well-defined electric susceptibility and magnetic permeability that, in many systems with complex geometries, are only approximately achieved. ${ }^{27}$

The effects of inhomogeneous broadening have previously been examined, e.g., by Gorkunov et al. on the bulk properties of left-handed materials in periodic infinite lattices, ${ }^{28}$ and have been experimentally observed by Gollub et al. ${ }^{29}$ In our study of ASR resonators, we evaluate collective modes of a finite lattice in order to investigate the effects of the inhomogeneous broadening on the experimentally observed sample sizedependent transmission resonant linewidth narrowing. ${ }^{1}$

In our analysis, we employ a general theoretical formalism of collective interactions between a discrete set of plasmonic resonators, or meta-atoms, mediated by the EM field that we developed in Ref. 4. In the model, we assume each meta-atom exhibits a single mode of current oscillation that possesses appropriate electric and magnetic dipole moments. Each meta-atom responds to EM fields exhibiting a specific resonance frequency and coupling strength that are determined by its characteristic design. Starting from the Lagrangian and Hamiltonian formalism describing the interaction of the EM field with polarization and magnetization densities created by a charge distribution, we then derived the coupled dynamics of the EM fields and the meta-atom dynamic variables. ${ }^{4}$ In a collection of meta-atoms, interactions with the EM field mediate a dynamic coupling between the meta-atoms and determine the collective dynamics within the ensemble, resulting in distinct collective modes with corresponding resonance frequencies and linewidths. The model of Ref. 4 has previously been successful in providing an excellent agreement between the theory and experimental observations of cooperative transmission resonance linewidth narrowing of ASR metamolecules. ${ }^{5}$

The analysis of collective response in terms of discrete resonators also points to the direction of an interesting analogy between resonators and a system of a cloud of atoms. In atoms, the electron transitions driven by an EM field create an electric dipole moment, while in the case of circuit elements, the oscillating current generates both the electric and magnetic dipole moments. The effects of inhomogeneous broadening due to thermal motion of atoms have been investigated in Dicke superradiance of atomic clouds (for a review, see Ref. 30, and for recent investigations in cold-atomic gases, e.g., Ref. 31). The superradiance is a consequence of collective modes in which an ensemble of emitters radiates more rapidly than an isolated emitter. The collective interactions in the metamaterial samples we study here, however, are notably more complex, as they not only involve electric-dipole scattering, but also magnetic-dipole scattering, which is absent in atomic systems.

The remainder of this article is organized as follows. We summarize the theoretical formalism we employ to describe collective interactions within the metamaterial ${ }^{4}$ in Sec. II. In Sec. III, we describe the fundamental building block of our metamaterical, the ASR, in the context of this formalism. The main results of the article, describing the effects of inhomogeneous broadening on the collective response of the metamaterial, are presented in Sec. IV, and conclusions follow in Sec. V.

\section{A MODEL FOR THE COLLECTIVE RESPONSE IN METAMATERIALS}

In order to incorporate the effects of strongly heterogeneous metamaterial, we describe the subwavelength structures of the medium as discrete scatterers. ${ }^{4}$ Each unit-cell element, a metamolecule, may also consist of subelements, which we call meta-atoms. While the general formalism of Ref. 4 allows for multipole-field radiation of the resonator unit elements, as a first approximation here we consider each subwavelength-sized meta-atom simply as a radiating dipole and ignore its multipole-field contribution. Following our treatment in Ref. 4 , we assume that each meta-atom $j$, with its position vector defined by $\mathbf{r}_{j}$, supports a single eigenmode of current oscillation. The dynamics of this current oscillation is determined by the dynamic variable $Q_{j}(t)$ with units of charge. Each meta-atom exhibits an electric- and magnetic-dipole moment. These may be expressed as

$$
\begin{aligned}
\mathbf{d}_{j} & =Q_{j} h_{j} \hat{\mathbf{d}}_{j}, \\
\mathbf{m}_{j} & =I_{j} A_{j} \hat{\mathbf{m}}_{j},
\end{aligned}
$$

respectively. Here, $I_{j}(t)=d Q_{j} / d t$ denotes the current, and the directions of the dipole moments are specified by the unit vectors $\hat{\mathbf{d}}_{j}$ and $\hat{\mathbf{m}}_{j}$ with proportionality coefficients $h_{j}$ and $A_{j}$ (with units of length and area, respectively) that depend on the specific geometry of the resonators. Although in this approximation each meta-atom possesses only electric and magnetic dipoles, a meta-molecule of two or more meta-atoms in our model would exhibit a nonvanishing quadrupole field. While, in general, this quadrupole contribution is inaccurately represented in the dipole approximation, in the case of the ASR metamolecules considered in Refs. 1 and 32 and in this study, the generated quadrupole field is notably suppressed when compared to the corresponding dipolar field. This has been indicated by finite element simulations of Maxwell's equations within a single metamolecule. ${ }^{33}$ Additionally, the fact that the size of the meta-atoms is often comparable to the spacing between them could result in a correction to the coupling strength between neighboring elements obtained in the point dipole approximation. Nonetheless, this model in the dipole approximation was employed in Ref. 5 to characterize the cooperative linewidth narrowing responsible for the enhancement of quality factor with system size observed in Ref. 1, yielding excellent agreement with experimental results. In this section, we present the key features of our theoretical formalism that are required to describe the collective response of an inhomogeneously broadened sample of ASRs to the EM field. Details of the derivation are presented in Ref. 4.

We write the polarization and magnetization densities as a sum of their contributions from the individual 
meta-atoms

$$
\begin{aligned}
\mathbf{P}(\mathbf{r}) & =\sum_{j} \mathbf{P}_{j}(\mathbf{r}), \\
\mathbf{M}(\mathbf{r}) & =\sum_{j} \mathbf{M}_{j}(\mathbf{r}),
\end{aligned}
$$

where the polarization and the magnetization of the resonator $j$ in the dipole approximation read as

$$
\begin{aligned}
\mathbf{P}_{j}(\mathbf{r}, t) & \approx \mathbf{d}_{j} \delta\left(\mathbf{r}-\mathbf{r}_{j}\right), \\
\mathbf{M}_{j}(\mathbf{r}, t) & \approx \mathbf{m}_{j} \delta\left(\mathbf{r}-\mathbf{r}_{j}\right),
\end{aligned}
$$

respectively.

An external beam with electric field $\mathbf{E}_{\text {in }}(\mathbf{r}, t)$ and magnetic field $\mathbf{H}_{\text {in }}(\mathbf{r}, t)$ with frequency $\Omega_{0}$ impinges on the ensemble of meta-atoms. The incident EM field drives the meta-atoms, generating dipole radiation from the oscillating electric and magnetic dipoles. The total radiation from the metamaterial array is the sum of the scattered electric and magnetic fields from all the meta-atoms:

$$
\begin{aligned}
& \mathbf{E}_{\mathrm{S}}(\mathbf{r}, t)=\sum_{j} \mathbf{E}_{\mathrm{S}, j}(\mathbf{r}, t), \\
& \mathbf{H}_{\mathrm{S}}(\mathbf{r}, t)=\sum_{j} \mathbf{H}_{\mathrm{S}, j}(\mathbf{r}, t),
\end{aligned}
$$

where $\mathbf{E}_{\mathrm{S}, j}(\mathbf{r}, t)$ and $\mathbf{H}_{\mathrm{S}, j}(\mathbf{r}, t)$ denote the electric and magnetic field emitted by the meta-atom $j$. The Fourier components of the scattered fields have the familiar expressions of electricand magnetic-dipole radiation, ${ }^{34}$

$$
\begin{aligned}
\mathbf{E}_{\mathrm{S}, j}^{+}(\mathbf{r}, \Omega)= & \frac{k^{3}}{4 \pi \epsilon_{0}} \int d^{3} r^{\prime}\left[\mathbf{G}\left(\mathbf{r}-\mathbf{r}^{\prime}, \Omega\right) \cdot \mathbf{P}_{j}^{+}\left(\mathbf{r}^{\prime}, \Omega\right)\right. \\
& \left.+\frac{1}{c} \mathbf{G}_{\times}\left(\mathbf{r}-\mathbf{r}^{\prime}, \Omega\right) \cdot \mathbf{M}_{j}^{+}\left(\mathbf{r}^{\prime}, \Omega\right)\right], \\
\mathbf{H}_{\mathrm{S}, j}^{+}(\mathbf{r}, \Omega)= & \frac{k^{3}}{4 \pi} \int d^{3} r^{\prime}\left[\mathbf{G}\left(\mathbf{r}-\mathbf{r}^{\prime}, \Omega\right) \cdot \mathbf{M}_{j}^{+}\left(\mathbf{r}^{\prime}, \Omega\right)\right. \\
& \left.-c \mathbf{G}_{\times}\left(\mathbf{r}-\mathbf{r}^{\prime}, \Omega\right) \cdot \mathbf{P}_{j}^{+}\left(\mathbf{r}^{\prime}, \Omega\right)\right],
\end{aligned}
$$

where $k \equiv \Omega / c$, and we have defined the positive and negative frequency components of a real time varying quantity $V(t)$ such that for a Fourier component of frequency $\Omega, V^{ \pm}(\Omega) \equiv$ $\Theta( \pm \Omega) V(\Omega)$, and hence $V(t)=V^{+}(t)+V^{-}(t)$ with $V^{-}(t)=$ $\left[V^{+}(t)\right]^{*}$. Here, $\mathbf{G}$ denotes the radiation kernel representing the electric (magnetic) field observed at position $\mathbf{r}$ emitted from an electric (magnetic) dipole at position $\mathbf{r}^{\prime}{ }^{34}$ Similarly, $\mathbf{G}_{\times}(\mathbf{r}, \Omega)$ represents the radiation kernel for the magnetic (electric) field observed at position $\mathbf{r}$ of an electric- (magnetic-) dipole source at point $\mathbf{r}^{\prime} .34$

A meta-atom $j$ exhibits behavior similar to that of an $L C$ circuit with resonance frequency ${ }^{4}$

$$
\omega_{j} \equiv \frac{1}{\sqrt{L_{j} C_{j}}},
$$

where $C_{j}$ is an effective self-capacitance and the effective self-inductance is $L_{j}$. In this work, we consider an inhomogeneously broadened sample of $N$ ASRs. Each ASR $l$ $(l=1, \ldots, N)$ consists of two meta-atoms, the resonance frequencies $\omega_{j}(j=2 l-1,2 l)$ of which are distributed according to $\omega_{0} \pm \delta \omega+X_{l}$, where $X_{l}$ are independent identically distributed random variables. We assume the meta-atom resonance frequencies occupy a narrow band about $\omega_{0}$, i.e., $|\delta \omega|,\left|X_{l}\right| \ll \omega_{0}$. The oscillating electric and magnetic dipoles of an isolated meta-atom radiate energy at respective rates $\Gamma_{\mathrm{E}}$ and $\Gamma_{\mathrm{M}}{ }^{4}$

$$
\begin{gathered}
\Gamma_{\mathrm{E}, j} \equiv \frac{h_{j}^{2} C_{j} \omega_{j}^{4}}{6 \pi \epsilon_{0} c^{3}}, \\
\Gamma_{\mathrm{M}, j} \equiv \frac{\mu_{0} A_{j}^{2} \omega_{j}^{4}}{6 \pi c^{3} L_{j}},
\end{gathered}
$$

resulting in the scattered fields $\mathbf{E}_{\mathrm{S}, j}$ and $\mathbf{H}_{\mathrm{S}, j}$ [see Eqs. (5) and (6)]. For simplicity, we assume that these radiative emission rates $\Gamma_{\mathrm{E}}$ and $\Gamma_{\mathrm{M}}$ are independent of the resonator $j$ and that they are dominated by the meta-atom resonance frequencies, i.e., $\Gamma_{\mathrm{E}, j}, \Gamma_{\mathrm{M}, j} \ll \Omega_{0}$. We further assume that the resonance frequencies occupy a narrow bandwidth around the central frequency of the incident field.

The dynamics of current excitations in the meta-atom $j$ may then be described by $Q_{j}(t)$ [introduced in Eq. (1)] and its conjugate momentum $\phi_{j}(t)$ (with units of magnetic flux ${ }^{4}$ ). In terms of the positive frequency components, the equations of motion read as ${ }^{4}$

$$
\begin{aligned}
\dot{Q}_{j}^{+} & =\left(1-i \frac{\Gamma_{\mathrm{M}}}{\omega_{j}}\right) \frac{\phi_{j}^{+}}{L_{j}}-\frac{\mu_{0} A_{j}}{L_{j}} \hat{\mathbf{m}}_{j} \cdot \mathbf{H}_{j, \mathrm{ext}}^{+}\left(\mathbf{r}_{j}, t\right), \\
\dot{\phi}_{j}^{+} & =-\left(1-i \frac{\Gamma_{\mathrm{E}}}{\omega_{j}}\right) \frac{Q_{j}^{+}}{C_{j}}+h_{j} \hat{\mathbf{d}}_{j} \cdot \mathbf{E}_{j, \mathrm{ext}}^{+}\left(\mathbf{r}_{j}, t\right),
\end{aligned}
$$

where the fields generated externally to meta-atom $j$ that drive its dynamics $\mathbf{E}_{j \text {,ext }}^{+}(\mathbf{r}, t)$ and $\mathbf{H}_{j, \text { ext }}^{+}(\mathbf{r}, t)$ are produced by the sums of the corresponding incident fields and the fields scattered by all other meta-atoms in the metamaterial sample $\sum_{j^{\prime} \neq j} \mathbf{E}_{\mathrm{S}, j^{\prime}}^{+}(\mathbf{r}, t)$ and $\sum_{j^{\prime} \neq j} \mathbf{H}_{\mathrm{S}, j^{\prime}}^{+}(\mathbf{r}, t)$, respectively. The component of the external electric field $\mathbf{E}_{j \text {,ext }}$ oriented along the dipole direction $\hat{\mathbf{d}}_{j}$ provides a net external electromotive force $(\mathrm{EMF}) h_{j} \hat{\mathbf{d}}_{j} \cdot \mathbf{E}_{j \text {,ext }}^{+}\left(\mathbf{r}_{j}, t\right)$ which drives $\phi_{j}(t)$. Similarly, the component of the external magnetic field $\mathbf{H}_{j \text {, ext }}$ along the magnetic dipole direction $\hat{\mathbf{m}}_{j}$ provides a net applied magnetic flux $A_{j} \mu_{0} \hat{\mathbf{m}}_{j} \cdot \mathbf{H}_{j, \text { ext }}^{+}\left(\mathbf{r}_{j}, t\right)$ that drives $Q_{j}(t)$. In the absence of radiative emission and interactions with external fields, current and charge oscillate within the meta-atom at the resonance frequency $\omega_{j}$. The meta-atom dynamics are therefore naturally described by the slowly varying normal variables

$$
b_{j}(t) \equiv \frac{e^{i \Omega_{0} t}}{\sqrt{2}}\left(\frac{Q_{j}(t)}{\sqrt{\omega_{j} C_{j}}}+i \frac{\phi_{j}(t)}{\sqrt{\omega_{j} L_{j}}}\right) .
$$

Without external field interactions and damping, $b_{j}$ oscillates with frequency $\left(\omega_{j}-\Omega_{0}\right)$, i.e., $b_{j}(t)=b_{j}(0) \exp \left[-i\left(\omega_{j}-\right.\right.$ $\left.\left.\Omega_{0}\right) t\right]$. For nonzero $\Gamma_{\mathrm{E}}, \Gamma_{\mathrm{M}} \ll \Omega_{0}$, losses and driving from the external field act to perturb this oscillation.

The current oscillation dynamics in the meta-atom $j$, described by $Q_{j}(t)$ and $\phi_{j}(t)$ in Eqs. (10) and (11), is driven by the incident field and the fields scattered from all the other meta-atoms and acts as a source of radiation that, in turn, drives the other meta-atoms. The expressions for the scattered fields by polarization and magnetization densities [Eqs. (5) 
and (6)] (generated by excitations in meta-atoms) and the expressions for the oscillating charge dynamics [Eqs. (10) and (11)] form a coupled set of equations, describing EM field mediated interactions between the resonators. In terms of the normal variables $b_{j}$, these interactions may be represented by the set of equations ${ }^{4}$

$$
\dot{\mathrm{b}}=\mathcal{C} \mathrm{b}+\mathrm{f}_{\mathrm{in}},
$$

where we have defined

$$
\mathrm{b}(t) \equiv\left(\begin{array}{c}
b_{1}(t) \\
b_{2}(t) \\
\vdots \\
b_{n N}(t)
\end{array}\right), \quad \mathrm{f}_{\mathrm{in}}(t) \equiv\left(\begin{array}{c}
f_{1, \text { in }}(t) \\
f_{2, \text { in }}(t) \\
\vdots \\
f_{n N, \text { in }}(t)
\end{array}\right) .
$$

The driving $f_{j \text {,in }}$ of each meta-atom $j$ results from the EMF and magnetic flux induced by the incident fields. ${ }^{4}$ Here, we assume that the meta-atom magnetic dipoles are aligned perpendicular to the incident magnetic field, and thus only the EMF contributes to the driving of each meta-atom, which is given by

$$
e^{-i \Omega_{0} t} f_{j, \text { in }}(t)=i \frac{h_{j}}{\sqrt{2 \omega_{j} L_{j}}} \hat{\mathbf{d}}_{j} \cdot \mathbf{E}_{\mathrm{in}}^{+}\left(\mathbf{r}_{j}, t\right) .
$$

The coupling matrix between the meta-atoms in Eq. (13) reads as

$$
\mathcal{C}=-i \Delta-\frac{\Gamma}{2} \mathrm{I}+\frac{1}{2}\left[i \Gamma_{\mathrm{E}} \mathcal{G}_{\mathrm{E}}+i \Gamma_{\mathrm{M}} \mathcal{G}_{\mathrm{M}}+\bar{\Gamma}\left(\mathcal{G}_{\times}+\mathcal{G}_{\times}^{T}\right)\right],
$$

where I represents the identity matrix, and $\bar{\Gamma} \equiv \sqrt{\Gamma_{\mathrm{E}} \Gamma_{\mathrm{M}}}$. Here, the detunings of the incident field from the meta-atom resonances are contained in the diagonal matrix $\Delta$ with elements

$$
\Delta_{j, j^{\prime}} \equiv \delta_{j, j^{\prime}}\left(\omega_{j}-\Omega_{0}\right),
$$

and the energy carried away from individual meta-atoms by the scattered fields manifests itself in the decay rate

$$
\Gamma \equiv \Gamma_{\mathrm{E}}+\Gamma_{\mathrm{M}}+\Gamma_{\mathrm{O}}
$$

appearing in the diagonal elements of $\mathcal{C}$. We account for nonradiative, e.g., Ohmic losses, by introducing phenomenological decay rate $\Gamma_{\mathrm{O}}$. The multiple scattering processes are included in the terms $\mathcal{G}_{\mathrm{E}}, \mathcal{G}_{\mathrm{M}}$, and $\mathcal{G}_{\times}$, which generate interaction between the meta-atom dynamic variables. The matrices $\mathcal{G}_{\mathrm{E}}$ and $\mathcal{G}_{\mathrm{M}}$ characterize the electric-dipole-dipole and magnetic-dipole-dipole interactions, respectively. Additionally, the interaction embodied by $\mathcal{G}_{x}$ arises from the electric field emitted by the magnetic dipole of one atom driving the electric dipoles of the others. Similarly, $\mathcal{G}_{\times}^{T}$ results from the magnetic field produced by the meta-atoms' electric dipoles impinging on the magnetic dipoles of all the other meta-atoms. The precise forms of $\mathcal{G}_{\mathrm{E}}, \mathcal{G}_{\mathrm{M}}$, and $\mathcal{G}_{\times}$are given in Ref. 4.

\section{ASYMMETRIC SPLIT-RING RESONATORS}

In order to investigate the effects of inhomogeneous broadening of meta-atom resonance frequencies on a metamaterial's collective EM response, we consider an ensemble of asymmetric metamolecules arranged in a regular lattice. To facilitate our description of this EM response, in this section we summarize the behavior of a single ASR in the context of the model presented in Sec. II.

An ASR is a variation on the split-ring resonator used to produce bulk metamaterials with negative indices of refraction. ${ }^{24-26}$ The meta-atoms of an ASR consist of two separate concentric circular arcs labeled by $j \in\{1, \mathrm{r}\}$ and separated by $\mathbf{u} \equiv \mathbf{r}_{\mathrm{r}}-\mathbf{r}_{1}$. The current oscillations in metaatoms produce electric dipoles with orientation $\hat{\mathbf{d}}_{\mathrm{r}}=\hat{\mathbf{d}}_{\mathrm{l}}=\hat{\mathbf{d}}$ associated with charge oscillating between the ends of the arcs. Owing to the curvature of the meta-atoms, these currents also produce magnetic dipoles with opposite orientations $\hat{\mathbf{m}}_{\mathrm{r}}=-\hat{\mathbf{m}}_{\mathrm{l}}=\hat{\mathbf{m}}$ where $\hat{\mathbf{d}} \perp \mathbf{u}$ and $\hat{\mathbf{m}} \perp \mathbf{u}, \hat{\mathbf{d}}$. An asymmetry between the rings, e.g., resulting from a difference in arc length, manifests itself as a difference in resonance frequencies with

$$
\begin{aligned}
& \omega_{\mathrm{r}}=\omega_{0}+\delta \omega, \\
& \omega_{1}=\omega_{0}-\delta \omega .
\end{aligned}
$$

To analyze the dynamics of a single ASR unit-cell resonator consisting of two meta-atoms, we apply the formalism presented in Sec. II. According to (13), the normal variables $b_{\mathrm{r}}$ and $b_{1}$ that describe the current oscillations in the right and left meta-atoms, respectively, are coupled by the EM fields so that they evolve according to

$$
\left(\begin{array}{c}
\dot{b}_{r} \\
\dot{b}_{l}
\end{array}\right)=\mathcal{C}^{\text {(ASR) }}\left(\begin{array}{c}
b_{r} \\
b_{l}
\end{array}\right)+\left(\begin{array}{c}
f_{r, \text { in }} \\
f_{l, \text { in }}
\end{array}\right) .
$$

Here, $\mathcal{C}^{(\mathrm{ASR})}$ denotes the specific coupling matrix between the two meta-atoms that depends of the radiative electricdipole-electric-dipole, magnetic-dipole-magnetic-dipole, and electric-dipole-magnetic-dipole interaction processes between the two meta-atoms [see Eq. (16)]. On the other hand, the incident field produces the driving terms $f_{j \text {,in }}$ for each meta-atom $j=l, r$ [see Eq. (15)].

To analyze the modes of the ASR, we consider the dynamics of symmetric $c_{+}$and antisymmetric $c_{-}$modes of oscillation defined by

$$
c_{ \pm} \equiv \frac{1}{\sqrt{2}}\left(b_{\mathrm{r}} \pm b_{1}\right) .
$$

The oscillations $c_{ \pm}$represent the eigenmodes of the ASR in the absence of asymmetry $\delta \omega=0$. By diagonalizing $\mathcal{C}^{(\mathrm{ASR})}$ with $\delta \omega=0$, one finds the eigenvalues of the modes $c_{ \pm}$:

$$
\lambda_{ \pm}=-i\left(\omega_{0}-\Omega_{0} \pm \Delta\right)-\frac{\gamma_{ \pm}}{2} .
$$

The interaction between the elements shifts the two collective resonance frequencies by equal and opposite amounts $\Delta$ and results in the decay rates $\gamma_{ \pm}$, where the coefficients $\Delta$ and $\gamma_{ \pm}$depend on the radiative interactions between the two meta-atoms. ${ }^{4,5}$ When the spacing between the arcs $u \ll \lambda$ $\left(\lambda=2 \pi c / \Omega_{0}\right)$, the decay rates simplify to

$$
\begin{aligned}
& \gamma_{+}=2 \Gamma_{\mathrm{E}}+\Gamma_{\mathrm{O}}, \\
& \gamma_{-}=2 \Gamma_{\mathrm{M}}+\Gamma_{\mathrm{O}} .
\end{aligned}
$$

In this limit, the symmetric mode, possessing a net electric dipole, emits electric-dipole radiation, and the antisymmetric mode, possessing a net magnetic dipole, emits magneticdipole radiation. We therefore may refer to symmetric and 
antisymmetric oscillations as electric- and magnetic-dipole excitations, respectively.

A nonzero asymmetry $\delta \omega \neq 0$ tends to couple the symmetric and antisymmetric oscillations. One finds that, when driven by an external field, these oscillations in a single ASR evolve as $^{4,5}$

$$
\dot{c}_{ \pm}=\left[-i\left(\omega_{0} \pm \Delta-\Omega_{0}\right)-\frac{\gamma_{ \pm}}{2}\right] c_{ \pm}-i \delta \omega c_{\mp}+F_{ \pm},
$$

where the driving terms $F_{ \pm}=\left(f_{\mathrm{r}, \text { in }} \pm f_{1, \text { in }}\right) / \sqrt{2}$. The symmetric and antisymmetric oscillations are driven purely by the electric and magnetic fields, respectively, and when the meta-atom separation $u \ll \lambda, F_{+} \propto \hat{\mathbf{d}} \cdot \mathbf{E}_{\text {in }}^{+}(\mathbf{R}, t)$ and $F_{-} \propto$ $\hat{\mathbf{m}} \cdot \mathbf{B}_{\text {in }}^{+}(\mathbf{R}, t)$, where $\mathbf{R}$ is the center of mass of the ASR. Therefore, an incident field with $\mathbf{E}_{\text {in }} \| \hat{\mathbf{d}}$ and $\mathbf{B}_{\text {in }} \perp \hat{\mathbf{m}}$ only excites the symmetric mode when $\delta \omega=0$. However, for $\delta \omega \neq$ 0 , the asymmetry couples the symmetric and antisymmetric modes, and this incident field can resonantly pump the antisymmetric magnetic mode via an effective two-photon transition. ${ }^{5}$

In this article, we consider a 2D metamaterial comprised of ASRs arranged in a regular array. The fields scattered from each ASR then mediate interactions between them, resulting in collective modes of oscillation distributed over the array, each with its own resonance frequency and decay rate. Figure 1 provides a schematic illustration of a mode consisting primarily of magnetic dipoles oscillating in phase throughout the metamaterial. ${ }^{4,5}$ We showed in Ref. 5 that, for sufficiently large array, such a mode radiates more slowly than the magnetic excitation of a single ASR and is responsible for the transmission resonance observed by Fedotov et al. ${ }^{1}$ When an incident EM field whose magnetic field is perpendicular to the ASR magnetic dipoles impinges on the array, this mode can not be excited directly. But, the presence of an asymmetry provides a coupling between electric and magnetic dipoles allowing it to be driven. Driving of the metamaterial's

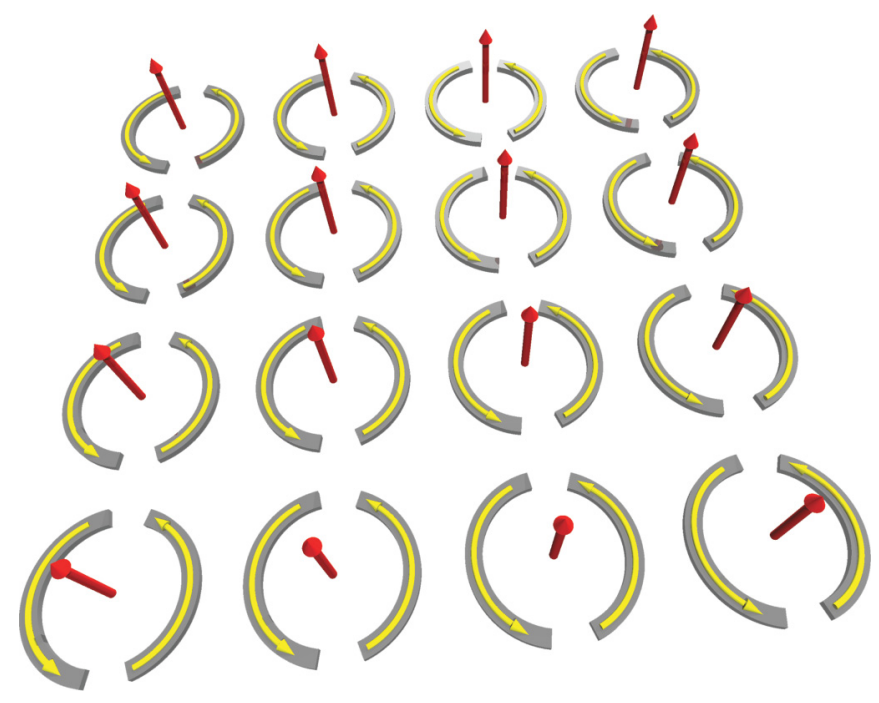

FIG. 1. (Color online) A schematic illustration of an array of ASR metamolecules excited in the uniform phase magnetic mode. The meta-atom currents in each ASR oscillate with opposite phases, producing magnetic dipoles represented by the red arrows. collective modes are responsible for the cooperative response that yields phenomena such as the observed transmission resonance. $^{1,5}$

\section{INHOMOGENEOUS BROADENING}

In this section, we study the effects of inhomogeneous broadening on the cooperative EM response of an array of ASRs. Here, inhomogeneous broadening refers to a statistical uncertainty in the resonance frequencies in individual ASR metamolecules. Such an uncertainty may result, for example, from imperfections in the manufacturing processes which yield meta-atoms, the shape of which varies slightly from the design specifications. Collective modes that are phase matched with an incident EM field have been shown to be responsible for transmission resonances ${ }^{5}$ that have been observed experimentally. ${ }^{1,32}$ We will illustrate the response of a regular array of ASRs to an incident plane wave, and show that broadening adversely affects the characteristic resonance behavior

We consider an ensemble of ASR metamolecules, the constituent meta-atoms of which are separated by $\mathbf{u}=u \hat{\mathbf{e}}_{x}$ with electric dipoles oriented along $\hat{\mathbf{d}}=\hat{\mathbf{e}}_{y}$ such that a symmetric oscillation in a single ASR produces an electric dipole along $\hat{\mathbf{d}}$, and an antisymmetric oscillation produces a magnetic dipole along $\hat{\mathbf{m}}=\hat{\mathbf{e}}_{z}$. The ASRs are arranged in an $N_{x} \times N_{y}$ 2D square lattice with lattice spacing $a$ and lattice vectors $\mathbf{a}_{1}=a \hat{\mathbf{e}}_{x}$ and $\mathbf{a}_{2}=a \hat{\mathbf{e}}_{y}$. The sample is illuminated by a cw plane wave $\mathbf{E}_{\text {in }}^{+}(\mathbf{r})=\frac{1}{2} \mathcal{E} \hat{\mathbf{e}}_{y} e^{i \mathbf{k} \cdot \mathbf{r}}$ with $\mathbf{k}=k \hat{\mathbf{e}}_{z}$, coupling exclusively to the electric-dipole moments of the ASRs.

The vector of $2 N_{x} N_{y}$ normal variables describing the state of current oscillations in each meta-atom obeys the coupled equations of motion in Eq. (13), where the matrix $\mathcal{C}$ [see Eq. (16)] arises from the meta-atom interactions mediated by the EM field. The metamaterial therefore exhibits $2 N_{x} N_{y}$ collective modes of oscillation corresponding to the eigenvectors $\mathrm{v}_{i}\left(i=1, \ldots, 2 N_{x} N_{y}\right)$ of interaction matrix $\mathcal{C}$. Each eigenmode $i$ possesses a particular resonance frequency $\Omega_{i}$ and decay rate $\gamma_{i}$ given in terms of the eigenvalue $\lambda_{i}$ by

$$
\begin{gathered}
\Omega_{i}=-\operatorname{Im}\left(\lambda_{i}\right)+\Omega_{0}, \\
\gamma_{i}=-2 \operatorname{Re}\left(\lambda_{i}\right),
\end{gathered}
$$

respectively.

Since the incident field drives all ASRs uniformly, it couples most effectively to the collective modes in which all of the metamolecules oscillate in phase. The two modes of particular interest are the uniform electric and uniform magnetic modes. In the absence of an asymmetry $(\delta \omega=0)$, the incident field drives the uniform electric mode which, owing to the electric-dipole orientations, emits strongly into the $\pm \hat{\mathbf{e}}_{z}$ directions. The electric dipoles oscillating in phase are responsible for reflection from the metamaterial. By contrast, the magnetic dipoles in the uniform magnetic mode, illustrated in Fig. 1, emit into the plane of the metamaterial array, and for sufficiently large lattices of closely spaced ASRs, the magnetic-dipole radiation becomes trapped. This results in a suppressed radiative decay rate of the uniform magnetic mode. ${ }^{5}$ Introduction of an asymmetry $(\delta \omega \neq 0)$ provides an effective coupling between these two collective modes similar 
to the coupling between the symmetric and antisymmetric modes in a single ASR. The collective magnetic mode can thus be resonantly excited at the expense of the electric dipoles, resulting in a transmission resonance, ${ }^{5,32}$ the quality factor of which increases with the size of the array, as observed by Fedotov et al. ${ }^{1}$

Specifically, the uniform magnetic mode is the eigenmode $\mathrm{v}_{\mathrm{m}}$ of $\mathcal{C}$ [see Eq. (16)] which maximizes the overlap $O_{\mathrm{m}}\left(\mathrm{b}_{A}\right)$ with the pure magnetic excitation $b_{\mathrm{A}}$ in which all meta-atoms are excited with equal amplitude and the two meta-atoms in each split ring oscillate out of phase. Similarly, the uniform electric mode is the eigenvector $\mathrm{v}_{\mathrm{e}}$ of $\mathcal{C}$ that maximizes the overlap $O_{\mathrm{e}}\left(\mathrm{b}_{\mathrm{S}}\right)$ with the pure electric excitation $\mathrm{b}_{\mathrm{S}}$ for which all current oscillations oscillate in phase with equal amplitude. Explicitly, these column vectors of $2 N_{x} N_{y}$ elements are given by

$$
\mathrm{b}_{\mathrm{A}} \equiv\left(\begin{array}{c}
+1 \\
-1 \\
\vdots \\
+1 \\
-1
\end{array}\right), \quad \mathrm{b}_{\mathrm{S}} \equiv\left(\begin{array}{c}
1 \\
1 \\
\vdots \\
1 \\
1
\end{array}\right)
$$

The alternating signs of the elements of $b_{A}$ indicate the relative phase of the oscillations in each meta-atom of an ASR. We define the overlap of mode $\mathrm{v}_{\mathrm{m} / \mathrm{e}}$ with an arbitrary excitation $\mathrm{b}$ as

$$
O_{\mathrm{m} / \mathrm{e}}(\mathrm{b}) \equiv \frac{\left|\mathrm{v}_{\mathrm{m} / \mathrm{e}}^{T} \mathrm{~b}\right|^{2}}{\sum_{i}\left|\mathrm{v}_{i}^{T} \mathrm{~b}\right|^{2}},
$$

where the index $i$ is summed over all the eigenmodes of the interaction matrix $\mathcal{C}$. The uniform electric and uniform magnetic modes for a $21 \times 21$ array of ASRs with a nonzero asymmetry parameter $\delta \omega=0.3 \Gamma$ are shown in Figs. 2 and 3 , respectively. We used the experimental value for the lattice spacing from Ref. 1 and estimate the asymmetry parameter $\delta \omega \simeq 0.3 \Gamma$ from the relative arc lengths of the ASR meta-atoms studied by Fedotov et al. ${ }^{1,32}$ The Ohmic loss rate $\Gamma_{\mathrm{O}}$ was fitted so that the quality factor of the uniform magnetic mode as a function of system size matched the experimental observations. ${ }^{5}$ Where the state of the ensemble is characterized by the vector of meta-atom normal variables $\mathrm{b}$ [see Eq. (14)], the symmetric (electric) and antisymmetric (magnetic) oscillations of an ASR $l\left(l=1, \ldots, N_{x} N_{y}\right)$ are represented by $c_{+, l}$ and $c_{-, l}$, respectively, where

$$
c_{ \pm, l} \equiv \frac{1}{\sqrt{2}}\left(b_{2 l-1} \pm b_{2 l}\right) \text {. }
$$

The respective symmetric (electric-dipole) and antisymmetric (magnetic-dipole) excitation energies in ASR $l$ are proportional to $\left|c_{+, l}\right|^{2}$ and $\left|c_{-, l}\right|^{2}$. The asymmetry in the ASRs causes a mixing of the electric and magnetic dipoles, producing a slight electric dipole excitation in the uniform magnetic mode $\mathrm{v}_{\mathrm{m}}$ and a slight magnetic excitation of the electric mode $\mathrm{v}_{\mathrm{e}}$. In the example illustrated in Figs. 2 and 3, when the Ohmic loss rate in each meta-atom is $\Gamma_{\mathrm{O}} \simeq 0.14 \Gamma$, the electric mode has an enhanced decay rate $\gamma_{\mathrm{e}}=2.7 \Gamma$ and the magnetic mode has a suppressed decay rate $\gamma_{\mathrm{m}}=0.31 \Gamma$ with respect to the total isolated single meta-atom decay rate $\Gamma$. Due to the suppressed
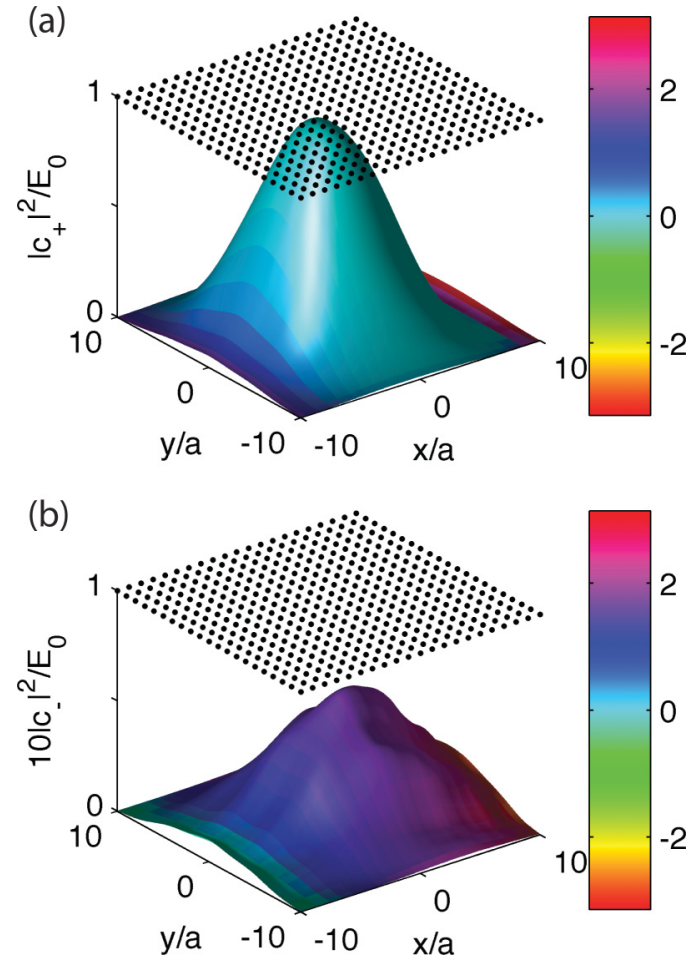

FIG. 2. (Color online) The uniform electric mode $v_{\mathrm{e}}$ of $a$ homogeneously broadened $(\sigma=0) 21 \times 21$ ASR square lattice. (a) The electric-dipole excitations $\left|c_{+, l}\right|^{2}$ and (b) the magnetic-dipole excitations $\left|c_{-, l}\right|^{2}$ of the uniform electric mode $\mathrm{v}_{\mathrm{e}}$. The phases of the electric- $\left(c_{+, l}\right)$ and magnetic- $\left(c_{-, l}\right)$ dipole excitations are indicated by the color of the surfaces in (a) and (b), respectively. The black dots indicate the positions of the ASRs in the array. The excitations $\left|c_{ \pm, l}\right|^{2}$ are normalized to the peak ASR excitation $E_{0}=$ $\max _{l}\left(\left|c_{+, l}\right|^{2}+\left|c_{-, l}\right|^{2}\right)$. The vertical scale of panel (b) was amplified by a factor of 10 to render the magnetic-dipole excitations $\left|c_{-, l}\right|^{2}$ visible. The vertices on the plots correspond to the ASR positions. The lattice spacing $a=0.28 \lambda$, meta-atom separation within an ASR $u=0.12 \lambda, \Gamma_{\mathrm{E}}=\Gamma_{\mathrm{M}}$, and asymmetry parameter $\delta \omega=0.3 \Gamma$.

decay rate of $\mathrm{v}_{\mathrm{m}}$ and its small electric-dipole component, this mode can be resonantly excited by the incident field.

We illustrate this phenomenon in Fig. 4, where we show the steady-state response [see Eq. (13)]

$$
\mathrm{b}_{\mathrm{r}} \equiv-\mathcal{C}^{-1} \mathrm{f}_{\mathrm{in}}
$$

of an array, the resonance frequencies of which are not inhomogeneously broadened. The right and left meta-atoms of ASR $l$ in such an array have respective resonance frequencies $\omega_{2 l-1}=\omega_{0}+\delta \omega$ and $\omega_{2 l}=\omega_{0}-\delta \omega$ centered around $\omega_{0}$. The driving field is resonant on the uniform magnetic mode, and the asymmetry in the split rings $\delta \omega=0.3 \Gamma$ facilitates the phase-coherent excitation of the magnetic dipoles at the expense of the electric dipoles. Figures 4(a) and 4(c) illustrate that the magnetic dipoles are much more strongly excited than the electric dipoles in the bulk of the array, and that these magnetic dipoles oscillate in phase. The more excited of the weak electric dipoles also oscillate in phase, thus facilitating the driving of this excitation by the uniform incident field. Although other collective modes of the system are excited, more than $60 \%$ of the excitation energy resides in the uniform 

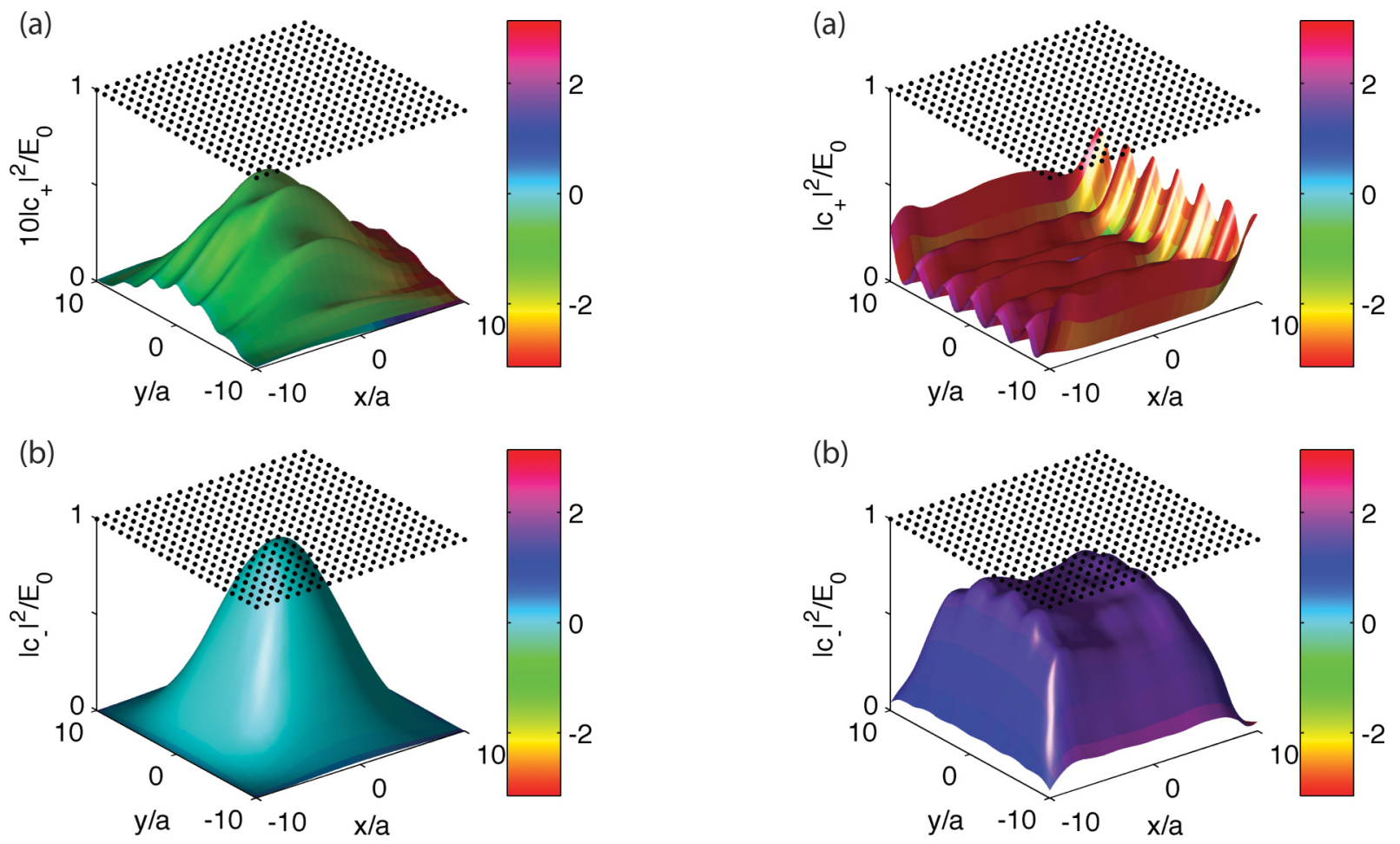

FIG. 3. (Color online) The uniform magnetic mode $v_{m}$ of a homogeneously broadened $(\sigma=0) 21 \times 21$ ASR square lattice. (a) The electric-dipole excitations $\left|c_{+, l}\right|^{2}$ and (b) the magnetic-dipole excitations $\left|c_{-, l}\right|^{2}$ of the uniform electric mode $\mathrm{v}_{\mathrm{e}}$. The phases of the electric- $\left(c_{+, l}\right)$ and magnetic- $\left(c_{-, l}\right)$ dipole excitations are indicated by the color of the surfaces in (a) and (b), respectively. The black dots indicate the positions of the ASRs in the array. The vertical scale of (a) was amplified by a factor of 10 to render the electric-dipole excitations $\left|c_{+, l}\right|^{2}$ visible. All parameters are as in Fig. 2.

magnetic mode $\mathrm{v}_{\mathrm{m}}$. In the absence of Ohmic losses, one can optimize the asymmetry parameter $\delta \omega$ in large lattices so that over $98 \%$ of the excitation energy resides in the uniform magnetic mode. ${ }^{5}$

The introduction of inhomogeneous broadening alters the collective interactions and can destroy the characteristics of the metamaterial response that produces the transmission resonance. We model the inhomogeneous broadening by shifting the central resonance frequency of each ASR $l$ by independent identically distributed Gaussian random variables $X_{l}$ with zero mean and standard deviation $\sigma$. With the asymmetry characterized by $\delta \omega$, the right and left circular arcs in ASR $l$ possess resonance frequencies $\omega_{2 l-1}=\omega_{0}+X_{l}+\delta \omega$ and $\omega_{2 l}=\omega_{0}+X_{l}-\delta \omega$, respectively. The deleterious effects of this broadening on the response are illustrated in Fig. 5, which shows a much less uniform magnetic response in addition to localized electric-dipole excitations. This nonuniformity inhibits the coherent reflection and transmission through the metamaterial array.

We quantify the effects of inhomogeneous broadening by examining the overlap of the metamaterial steady-state response $b_{r}$ with the magnetic mode $v_{m}(\sigma=0)$ of a homogeneously broadened array (corresponding to the case in which all ASR metamolecules are identical). If the excitation is purely in the mode $\mathrm{v}_{\mathrm{m}}(0)$, then the overlap $O_{\mathrm{m}}\left(\mathrm{b}_{\mathrm{r}}\right)$ [Eq. (28)]

FIG. 4. (Color online) The response of a homogeneously broadened $(\sigma=0) 21 \times 21$ ASR square lattice to an incident plane-wave electric field resonant on the uniform collective magnetic mode $\mathrm{v}_{\mathrm{m}}$, showing the excitation of a uniform magnetic-dipole excitation at the expense of electric dipoles. (a) The electric-dipole excitations $\left|c_{+, l}\right|^{2}$ and (b) the magnetic-dipole excitations $\left|c_{-, l}\right|^{2}$ of the response. The phases of the electric- $\left(c_{+, l}\right)$ and magnetic- $\left(c_{-, l}\right)$ dipole excitations are indicated by the color of the surfaces in (a) and (b), respectively. The black dots indicate the positions of the ASRs in the array. The Ohmic loss rate is $\Gamma_{\mathrm{O}}=0.14 \Gamma$. All other parameters are as in Fig. 2.

is unity. Figure 6(a) shows the overlap $O_{\mathrm{m}}\left(\mathrm{b}_{\mathrm{r}}\right)$ of the response to an incident field resonant on the homogeneously broadened mode $\mathrm{v}_{\mathrm{m}}(\sigma=0)$ averaged over 240 realizations. The solid black line was calculated for the same parameters as in Figs. 2 through 5 with varying degrees of broadening. The blue dashed curve shows the corresponding overlap in the absence of Ohmic losses, while for the red dashed-dotted curve, the asymmetry parameter was reduced to $\delta \omega=0.1$ and $\Gamma_{\mathrm{O}}=0$. In all cases, as the broadening standard deviation $\sigma$ becomes comparable to $\delta \omega$, the ability to excite the uniform magnetic mode drastically decreases. The other modes that are excited either contain electric-dipole components or are not phase matched. This either results in scattering of the field or in absorption of the field due to Ohmic losses. As a result, the coherent collective response responsible for the transmission resonance observed in by Fedotov et al. ${ }^{1}$ becomes unobservable when the inhomogeneous broadening is larger than $\delta \omega$. These collective effects do persist, however, for $\sigma$ roughly half $\delta \omega$. We show the effect of inhomogeneous broadening in the decay rate $\gamma_{\mathrm{m}}(\sigma)$ of the magnetic mode itself in Fig. 6(b) as a function of broadening. Randomization of the ASR resonance frequencies apparently has little effect on the collective linewidth of the magnetic mode for $\sigma<$ $\delta \omega$. However, for larger degrees of broadening, the decay 

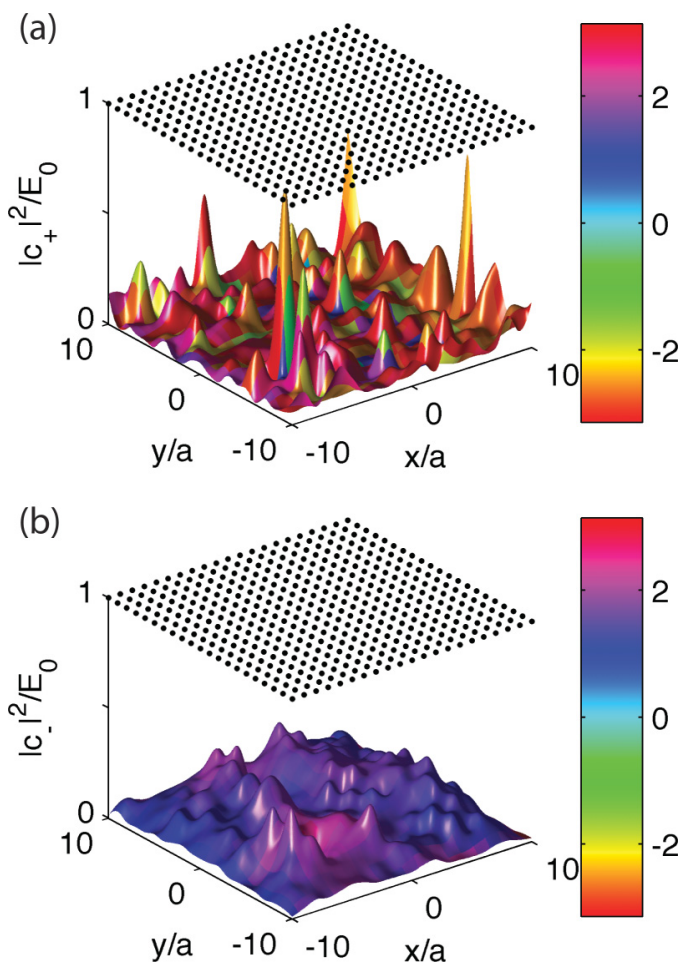

FIG. 5. (Color online) Response of inhomogeneously broadened square lattice to an incident plane wave resonant on the homogeneously broadened uniform magnetic mode $\mathrm{v}_{\mathrm{m}}$. (a) The electricdipole excitations $\left|c_{+, l}\right|^{2}$ and (b) the magnetic-dipole excitations $\left|c_{-, l}\right|^{2}$ of the response. The phases of the electric- $\left(c_{+, l}\right)$ and magnetic$\left(c_{-, l}\right)$ dipole excitations are indicated by the color of the surfaces in (a) and (b), respectively. The black dots indicate the positions of the ASRs in the array. The individual ASR resonance frequencies are shifted by independent identically distributed Gaussian random variables with standard deviation $\sigma=0.8 \delta \omega$. All other parameters are as in Fig. 4.

rate of this mode can be increased several times over and the resonance linewidth narrowing, which results from the cooperative response of the metamaterial array, disappears. Furthermore, the large standard deviations of $\gamma_{\mathrm{m}}(\sigma)$ indicate that the width of the uniform magnetic mode is highly sensitive to the particular realization of ASR resonance frequencies. A larger decay rate renders the magnetic mode more difficult to excite since any excitation of this mode is more quickly radiated away. In order for an array of ASRs to exhibit a transmission resonance, a large fraction of the excitation created by the driving field must be in the uniform magnetic mode distributed over the array. In low-loss metamaterials, this can be achieved in conjunction with a higher quality for that resonance for larger arrays and smaller values of $\delta \omega .^{5}$ However, as Fig. 6 indicates, reduction in the asymmetry to achieve this quality-factor enhancement correspondingly reduces the tolerance for inhomogeneous broadening in the resonance frequency.

The observation that the cooperative metamaterial response to EM fields can be suppressed in the presence of sufficiently strong inhomogeneous broadening could potentially also be exploited in design of metamaterial samples that would benefit from well-defined homogeneous properties for
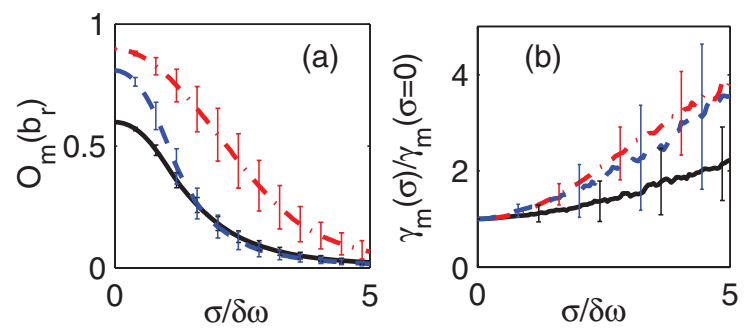

FIG. 6. (Color online) (a) The overlap of the uniform magnetic mode with the excitation driven by an incident EM plane wave and (b) the decay rate $\gamma_{\mathrm{m}}(\sigma)$ of the uniform magnetic mode $\mathrm{v}_{\mathrm{m}}(\sigma)$ as a function of inhomogeneous broadening $\sigma$. These quantities are plotted for $\delta \omega=0.3 \Gamma$ and Ohmic losses $\Gamma_{\mathrm{O}}=0.14 \Gamma$ (solid black line), $\delta \omega=0.3 \Gamma$ and $\Gamma_{\mathrm{O}}=0$ (dashed blue line), and $\delta \omega=0.1 \Gamma$ and $\Gamma_{\mathrm{O}}=0$ (dotted-dashed red line). The lines indicate the average over 240 sample realizations, and the error bars indicate the standard deviations. The incident wave has electric field polarization $\hat{\mathbf{e}}_{y}$ aligned with the ASR electric dipoles and is resonant on the mode $\mathrm{v}_{\mathrm{m}}(\sigma=0)$ of the homogeneously broadened array. The decay rates of the uniform magnetic modes for a homogeneously broadened array $\gamma_{\mathrm{m}}(\sigma=0)$ are $0.308 \Gamma$ (black line), $0.145 \Gamma$ (blue line), and $0.0371 \Gamma$ (red line). This shows that excitation of the magnetic mode, and hence the transmission resonance, vanishes as the inhomogeneous broadening becomes comparable to $\delta \omega$.

electric susceptibility and magnetic permeability, such as diffraction-free lenses due to negative refractive index. ${ }^{24-26}$ One could prepare a controlled amount of inhomogeneous broadening for the metamaterial sample in order to generate an EM response that more closely mimics standard continuous medium electrodynamics with suppressed contribution from recurrent scattering events.

\section{CONCLUSION}

In conclusion, we have analyzed the collective modes of a finite-sized 2D metamaterial array of ASR resonators and how they are influenced by an inhomogeneous broadening of the resonance frequencies of the individual resonators. The study was motivated by recent experimental observations of transmission resonance linewidth narrowing as a function of the size of the system. ${ }^{1}$ This effect can be understood by analyzing the resonance linewidths of collective modes of the system that undergo dramatic narrowing due to strong EM field mediated interactions between the resonators. As demonstrated by previous comparisons between the numerical simulation results and the experimental observations, ${ }^{5}$ the response can be analyzed by a simplified model in which each meta-atom is treated as a discrete element supporting a single mode of current oscillation possessing electric- and magnetic-dipole moments. Collective interactions between the meta-atoms are mediated by the scattered EM fields.

We examined in detail how inhomogeneous broadening of resonator resonance frequencies impairs the coherent collective phenomena that are expected to find important applications in metamaterial systems. ${ }^{6,8,10,32}$ While the transmission resonance experimentally observed in Ref. 1 persists for inhomogeneous broadening that is a fraction of the ASR asymmetry parameter $\delta \omega$, the cooperative response vanishes when the 
broadening begins to exceed that parameter. Production of high-quality resonances with low-loss materials requires the reduction of $\delta \omega .^{5}$ Figure 6 indicates that, in order to produce such high-quality resonances, the uniformity in the production of metamolecules will need to become correspondingly small.

Introducing disorder in the metamolecule positions has also been observed to destroy the transmission resonance ${ }^{2}$ by altering the interactions between resonators. The disorder changes the cooperative response rather than eliminating it.
However, as with the regular lattice we examined here, we expect that inhomogeneous broadening would also suppress cooperative phenomena arising from a metamaterial with disordered metamolecule positions.

\section{ACKNOWLEDGMENT}

We acknowledge discussions with N. Papasimakis, V. Fedotov, and N. Zheludev and financial support from the EPSRC and the Leverhulme Trust.
${ }^{1}$ V. A. Fedotov, N. Papasimakis, E. Plum, A. Bitzer, M. Walther, P. Kuo, D. P. Tsai, and N. I. Zheludev, Phys. Rev. Lett. 104, 223901 (2010).

${ }^{2}$ N. Papasimakis, V. A. Fedotov, Y. H. Fu, D. P. Tsai, and N. I. Zheludev, Phys. Rev. B 80, 041102(R) (2009).

${ }^{3}$ S. Savo, N. Papasimakis, and N. I. Zheludev, Phys. Rev. B 85, 121104(R) (2012).

${ }^{4}$ S. D. Jenkins and J. Ruostekoski, Phys. Rev. B 86, 085116 (2012).

${ }^{5}$ S. D. Jenkins and J. Ruostekoski, New J. Phys. 14, 103003 (2012).

${ }^{6}$ N. I. Zheludev, S. L. Prosvirnin, N. Papasimakis, and V. A. Fedotov, Nat. Photonics 2, 351 (2008).

${ }^{7}$ G. Adamo, J. Y. Ou, J. K. So, S. D. Jenkins, F. De Angelis, K. F. MacDonald, E. Di Fabrizio, J. Ruostekoski, and N. I. Zheludev, Phys. Rev. Lett. 109, 217401 (2012).

${ }^{8}$ T. S. Kao, S. D. Jenkins, J. Ruostekoski, and N. I. Zheludev, Phys. Rev. Lett. 106, 085501 (2011).

${ }^{9}$ T. S. Kao, E. T. F. Rogers, J. Y. Ou, and N. I. Zheludev, Nano Lett. 12, 2728 (2012).

${ }^{10}$ A. Sentenac and P. C. Chaumet, Phys. Rev. Lett. 101, 013901 (2008).

${ }^{11}$ A. Ishimaru, Wave Propagation and Scattering in Random Media: Multiple Scattering, Turbulence, Rough Surfaces, and RemoteSensing (University of California, Academic Press, St. Louis, MO, 1978), Vol. 2.

${ }^{12}$ B. A. van Tiggelen, A. Lagendijk, and A. Tip, J. Phys.: Condens. Matter. 2, 7653 (1990).

${ }^{13}$ O. Morice, Y. Castin, and J. Dalibard, Phys. Rev. A 51, 3896 (1995).

${ }^{14}$ J. Ruostekoski and J. Javanainen, Phys. Rev. A 55, 513 (1997).

${ }^{15}$ J. Ruostekoski and J. Javanainen, Phys. Rev. A 56, 2056 (1997).

${ }^{16}$ J. Javanainen, J. Ruostekoski, B. Vestergaard, and M. R. Francis, Phys. Rev. A 59, 649 (1999).
${ }^{17}$ P. de Vries, D. V. van Coevorden, and A. Lagendijk, Rev. Mod. Phys. 70, 447 (1998).

${ }^{18}$ J. Ruostekoski and J. Javanainen, Phys. Rev. Lett. 82, 4741 (1999).

${ }^{19}$ C. A. Müller, T. Jonckheere, C. Miniatura, and D. Delande, Phys. Rev. A 64, 053804 (2001).

${ }^{20}$ F. A. Pinheiro, M. Rusek, A. Orlowski, and B. A. van Tiggelen, Phys. Rev. E 69, 026605 (2004).

${ }^{21}$ S. D. Jenkins and J. Ruostekoski, Phys. Rev. A 86, 031602(R) (2012).

${ }^{22}$ L. Chomaz, L. Corman, T. Yefsah, R. Desbuquois, and J. Dalibard, New J. Phys. 14, 055001 (2012).

${ }^{23}$ J. Kästel, M. Fleischhauer, and G. Juzeliūnas, Phys. Rev. A 76, 062509 (2007)

${ }^{24}$ D. R. Smith, W. J. Padilla, D. C. Vier, S. C. Nemat-Nasser, and S. Schultz, Phys. Rev. Lett. 84, 4184 (2000).

${ }^{25}$ R. A. Shelby, D. R. Smith, and S. Schultz, Science 292, 77 (2001).

${ }^{26}$ D. R. Smith, J. B. Pendry, and M. C. K. Wiltshire, Science 305, 788 (2004).

${ }^{27}$ Z. S. G.-H. Park, R. Hedge, and E.-P. Li, IEEE Trans. Microwave Theory Tech. 58, 2646 (2010).

${ }^{28}$ M. V. Gorkunov, S. A. Gredeskul, I. V. Shadrivov, and Y. S. Kivshar, Phys. Rev. E 73, 056605 (2006).

${ }^{29}$ J. Gollub, T. Hand, S. Sajuygbe, S. Mendonca, S. Cummer, and D. R. Smith, Appl. Phys. Lett. 91, 162907 (2007).

${ }^{30}$ M. Gross and S. Haroche, Phys. Rep. 93, 301 (1982).

${ }^{31}$ T. Bienaimé, N. Piovella, and R. Kaiser, Phys. Rev. Lett. 108, 123602 (2012)

${ }^{32}$ V. A. Fedotov, M. Rose, S. L. Prosvirnin, N. Papasimakis, and N. I. Zheludev, Phys. Rev. Lett. 99, 147401 (2007).

${ }^{33}$ N. Papasimakis (unpublished).

${ }^{34}$ J. D. Jackson, Classical Electrodynamics (Wiley, New York, 1998). 